\title{
Geysers in the Lagoon: new Herbig-Haro objects in M 8*
}

\author{
R. H. Barbá ${ }^{1, \star \star}$ and J. I. Arias ${ }^{1,2, \star \star \star}$ \\ 1 Departamento de Física, Universidad de La Serena, Benavente 980, La Serena, Chile \\ e-mail: rbarba@dfuls.cl \\ ${ }^{2}$ Facultad de Ciencias Astronómicas y Geofísicas, Universidad Nacional de La Plata, and Instituto de Astrofísica La Plata, \\ Paseo del Bosque S/N, B1900FWA, La Plata, Argentina \\ e-mail: julia@djuls.cl
}

Received 21 July 2006 / Accepted 15 June 2007

\begin{abstract}
Aims. We search for direct evidence of ongoing star formation by accretion in the Lagoon Nebula (M 8), using optical wide-field narrow-band imaging obtained at La Silla Observatory.

Methods. We examine [S II] and $\mathrm{H} \alpha$ images for line-emission features that could be interpreted as signatures of outflow activity of the exciting sources.

Results. We discover five new Herbig-Haro objects, study in detail their morphology and attempt to identify their potential driving sources among the population of T Tauri stars and embedded sources in the surroundings.

Conclusions. The results reported here conclusively demonstrate the existence of very young stars going through the accreting phase in the $\mathrm{M} 8$ region.
\end{abstract}

Key words. ISM: Herbig-Haro objects - ISM: jets and outflows - stars: formation

\section{Introduction}

Herbig-Haro objects are direct manifestations of the formation process of low- and intermediate-mass stars. At present there is strong evidence that accretion disks surround the exciting sources of these stellar jets (Reipurth \& Heathcote 1992; Reipurth \& Bally 2001; White et al. 2004; Stecklum et al. 2004; Kastner et al. 2005; Comerón \& Reipurth 2006) and that accretion energy powers outflows (Hartigan et al. 1994, 1995; Goodson et al. 1997; Turner et al. 1999).

The vast majority of the known $\mathrm{HH}$ objects belong to nearby star forming regions (distances $\leq 500 \mathrm{pc}$ from the Sun) and have typical sizes of a fraction of a parsec. In recent years, larger and more sensitive detectors made possible to extend the survey to more distant star forming regions and, more importantly, allowed the discovery of a new class of $\mathrm{HH}$ objects which stretch over parsec scales (Bally \& Devine 1994).

The Lagoon Nebula (Messier 8, NGC 6523-NGC 6530) is a prominent galactic $\mathrm{H}$ II region at a distance of $1.25 \mathrm{kpc}$ (Arias et al. 2006, and references therein). During the last years several studies significantly increased our knowledge of the young stellar content in the region. Specifically, Sung et al. (2000), Damiani et al. (2004), Prisinzano et al. (2005) and Arias et al. $(2006,2007)$ pointed out the presence of an abundant population of young stellar objects (YSOs) with typical ages of about $10^{6}$ years, distributed over the whole nebula. In spite of the youth suggested by many stars in M 8, direct evidence of YSOs going through the accretion phase remained elusive. The first

* Based on observations made with the MPG/ESO 2.2-m telescope at La Silla Observatory

$\star \star$ Member of the Carrera del Investigador Científico, CONICET, Argentina.

$\star \star \star$ Fellow of CONICET, Argentina. mention regarding the existence of $\mathrm{HH}$ objects in $\mathrm{M} 8$ was made by Reipurth (1981). More recently, Arias et al. (2006) reported the first spectroscopic confirmation of the HH 870 outflow, located in the Hourglass Nebula, very close to the ZAMS O-star Herschel 36. Additionally, Zhang et al. (2005) presented evidence of a molecular outflow driven by the luminous YSO M8E-IR.

In this paper we report the discovery of new optical $\mathrm{HH}$ outflows in M 8, which definitely demonstrate the existence of young stars transiting the accretion phase of their formation. The spectroscopic confirmation of the $\mathrm{HH}$ nature of some of these objects will be presented in a forthcoming paper.

\section{Observations}

The optical images of NGC 6530 used in this study were acquired with the Wide Field Imager (WFI) at the MPG/ESO 2.2-m telescope at La Silla Observatory. The observational set consists of five [S II] and five $\mathrm{H} \alpha$ exposures, each with a duration of $180 \mathrm{~s}$, and five $R$ images with an exposure time of $10 \mathrm{~s}$ each. The WFI camera is an array of $2 \times 4$ CCD chips which have $2048 \times 409615-\mu \mathrm{m}$ pixels each. The pixel scale is 0.238 pix $^{-1}$, giving a $34 \times 33$ square arcmin field of view. The images were obtained on 2000 March 17 (filters \#856, H $\alpha$ 16562, and \#857, [S II] $\lambda \lambda 6717,6731)$ under Program ID\# 2064.I-0559 and 2001 June 17 (filter $R_{\mathrm{c}}$ ) under Program ID\# 165.5-0187(A). Both data sets, along with their respective bias and flat-field frames for calibration, were retrieved from the ESO/ST-ECF Science Archive Facility. The data reduction was performed using the IRAF $^{1}$ mscred package, implemented by NOAO for specific processing of CCD mosaics. The images were bias subtracted

\footnotetext{
1 IRAF is distributed by NOAO, operated by AURA, Inc., under agreement with NSF.
} 
and flat-fielded following the standard procedure provided by the mscred user's manual and the WFI web page. For each filter, the images were combined into a final deeper mosaic free from gaps and artifacts. The FWHM of the PSFs are 1".2 and 1". 4 for the $[\mathrm{S}$ II $]$ and $\mathrm{H} \alpha$ frames, respectively. Because three of the $\mathrm{H} \alpha$ exposures were affected by tracking errors, $\sim 1 \%$ intensity ghost images are observed $2^{\prime \prime}$ to the west of each object in the final $\mathrm{H} \alpha$ frame. These secondary images are particularly noticeable for the brightest stars.

The images were not flux calibrated. In order to compare the [S II] and $\mathrm{H} \alpha$ images, we constructed the difference map between $\mathrm{H} \alpha$ and four times the [S II] image, which gives a good picture of how both emission lines are related.

Celestial coordinates for the images were obtained from the 2MASS Point Source Catalogue (Skrutskie et al. 2006), using the msccmatch task. Only objects with $K_{\mathrm{s}}<12$ and photometric quality flag " $A A A$ " were considered. In order to estimate the astrometric accuracy, we positionally matched the stars in our catalogue with those in both the 2MASS PSC and the Guide Star Catalogue Version 2.2.01 (GSC 2.2, STScI, 2001). The rms residuals between the positional tables from this work and from the former data bases are found to be $\sim 0{ }^{\prime} 4$ in both coordinates.

\section{Identification of new Herbig-Haro objects}

Figure 1 shows a three-colour image of the M 8 nebula, with $\mathrm{H} \alpha$ in blue, [S II] $\lambda \lambda 6717,6731$ in red, and [S II] $+\mathrm{H} \alpha$ in green. The image exhibits an extremely rich and complex structure. The visual inspection of the individual narrow-band images allowed us to distinguish several nebular features, such as ionization fronts, "fingers", dark and bright globules, etc. Some of them could be identified as $\mathrm{HH}$ objects.

The newly identified $\mathrm{HH}$ objects are primarily located in the southern edge of M 8, formed by a group of bright-rimmed clouds defined as "Southeastern Bright Rim" and "Extended Bright Rim” by Lada et al. (1976a). Tothill et al. (2002) resolved several continuum $(850 \mu \mathrm{m})$ and CO clumps in this area. The presence of several classical and weak $\mathrm{T}$ Tauri stars and Herbig Ae/Be objects was also reported (Arias et al. 2007; Sung et al. 2000, and references therein). The rectangles in Fig. 1 indicate the regions where the new $\mathrm{HH}$ flows are found. These objects are shown in detail in Figs. 2 to 6. Their equatorial coordinates and some related notes are given in Table 1.

In this section we describe the morphological properties of the new $\mathrm{HH}$ objects and present some speculation about their potential driving sources.

\section{1. $\mathrm{HH} 893$}

$\mathrm{HH} 893$ is a small object situated close to a set of bright $\mathrm{H} \alpha$ filaments. Detailed views of this object are shown in Fig. 2. HH 893 is barely detectable in the $\mathrm{H} \alpha$ and $R$ images. In the [S II] image, it is composed of two bright emission knots (A and $\mathrm{B}$ ) separated by $\sim 1^{\prime \prime}$. Its size is approximately $6^{\prime \prime} \times 4^{\prime \prime}(7500 \times 5000 \mathrm{AU}$ at the distance of M 8), the eastern lobe A being larger.

In order to investigate the emission characteristics of this object, we considered the ratio of $\mathrm{H} \alpha+[\mathrm{S} \mathrm{II}]$ to the $R$-band flux (bottom right panel of Fig. 2). If there were significant scattered light (from any existing continuum source), this ratio would depart from unity. However, the observed ratio is around unity, implying that the filaments that form $\mathrm{HH} 893$ are entirely emission knots.

We could not identify any convincing driving source for $\mathrm{HH}$ 893. As the M 8 nebula is seen projected against the galactic bulge, a dense population of background infrared sources is observed. The nearest 2MASS source is located 2 '. 2 to the west of the feature $B$ of HH 893, but its near-IR colours correspond to a typical reddened background giant $\left(J-H=2.04, H-K_{\mathrm{s}}=0.83\right)$. We note here that HH 893 is located $\sim 20^{\prime \prime}$ to the west of the $850 \mu$ clump C3 (Tothill et al. 2002) and that the nearest IRAS source (18008-2425) is about 2!7 distant (see Fig. 1).

\section{2. $\mathrm{HH} 894$}

HH 894 shows remarkable characteristics. In Fig. 3 we present three images showing different aspects of this object. HH 894 consists of various components, which are labeled A, B and C in this figure. The structures A and B consist of a set of filaments that curve to form bow-shaped features brightest at their tips. While feature A shows two extended parabolic tails, feature B appears much more diffusely. The component B may be in turn decomposed in three smaller knots. The working surfaces are more evident in the $\mathrm{H} \alpha$ image. Tracking back about $68^{\prime \prime}$ along the line joining the bright tips of the knots $\mathrm{A}$ and $\mathrm{B}$, a small [S II] linear feature arises. This $10^{\prime \prime}$ size knot is labeled $\mathrm{C}$ in Fig. 3. If features $\mathrm{A}, \mathrm{B}$, and $\mathrm{C}$ were part of the same outflow, then the projected size of this outflow would be of approximately $80^{\prime \prime}$, which corresponds to $\sim 0.5 \mathrm{pc}$ at the distance of $\mathrm{M} 8$.

Several faint optical and near-IR stars are found lying on the presumed axis of this jet. This presumed axis also intersects the tip of a dusty pillar structure known as clump SC8 (Tothill et al. 2002), where three emission line stars, ABM 20, ABM 22 (Arias et al. 2007) and LkH $\alpha 110$ (Herbig 1957), are located (see Fig. 1). ABM 20 and ABM 22 were recently identified as T Tauri stars. The latter is additionally a very particular object since it shows asymmetric forbidden emission lines, which might be a signature of $\mathrm{HH}$ outflows. This naturally leads to speculation that HH 894 is driven by some of the sources located at the tip of $\mathrm{SC} 8$, in which case its projected length would be of about $3^{\prime}$ or $1.1 \mathrm{pc}$ at the distance of M 8. Future spectroscopic studies will help to answer this question.

\section{3. $\mathrm{HH} 895$}

HH 895 shows a very peculiar morphology. In Fig. 4 we present three detailed views of this intriguing object. It consists of a large bow-shaped arc (A) with a central condensation (B). The arc, whose amplitude is of approximately $50^{\prime \prime}$, is dominated by $\mathrm{H} \alpha$ emission and hence appears whitish in the $\mathrm{H} \alpha-4 *[\mathrm{~S} \mathrm{II}]$ difference image shown in the bottom panel of Fig. 4. The component $\mathrm{B}$ is a curious rhomboidal structure, whose western edge looks brighter in [S II]. We note here that $\mathrm{HH} 895$ is found in a region where a complex network of filaments exists. A further analysis is needed to disentangle the several components present in this area.

\section{4. $\mathrm{HH} 896$}

Two nebular features are found roughly equally distant north and south from the $850 \mu$ clump SE3 identified by Tothill et al. (2002). The IRAS source 18014-2428 is also associated with this molecular clump (see Figs. 1 and 5). The northern feature, located about 2!2 from IRAS 18014-2428, is the HH 896 outflow.

The right panel of Fig. 6 presents a close-up of $\mathrm{HH} 896$ in the light of [S II]. This object consists of two well-defined parabolic bow shocks (A and B), along with a fainter nebular arc (C). The 


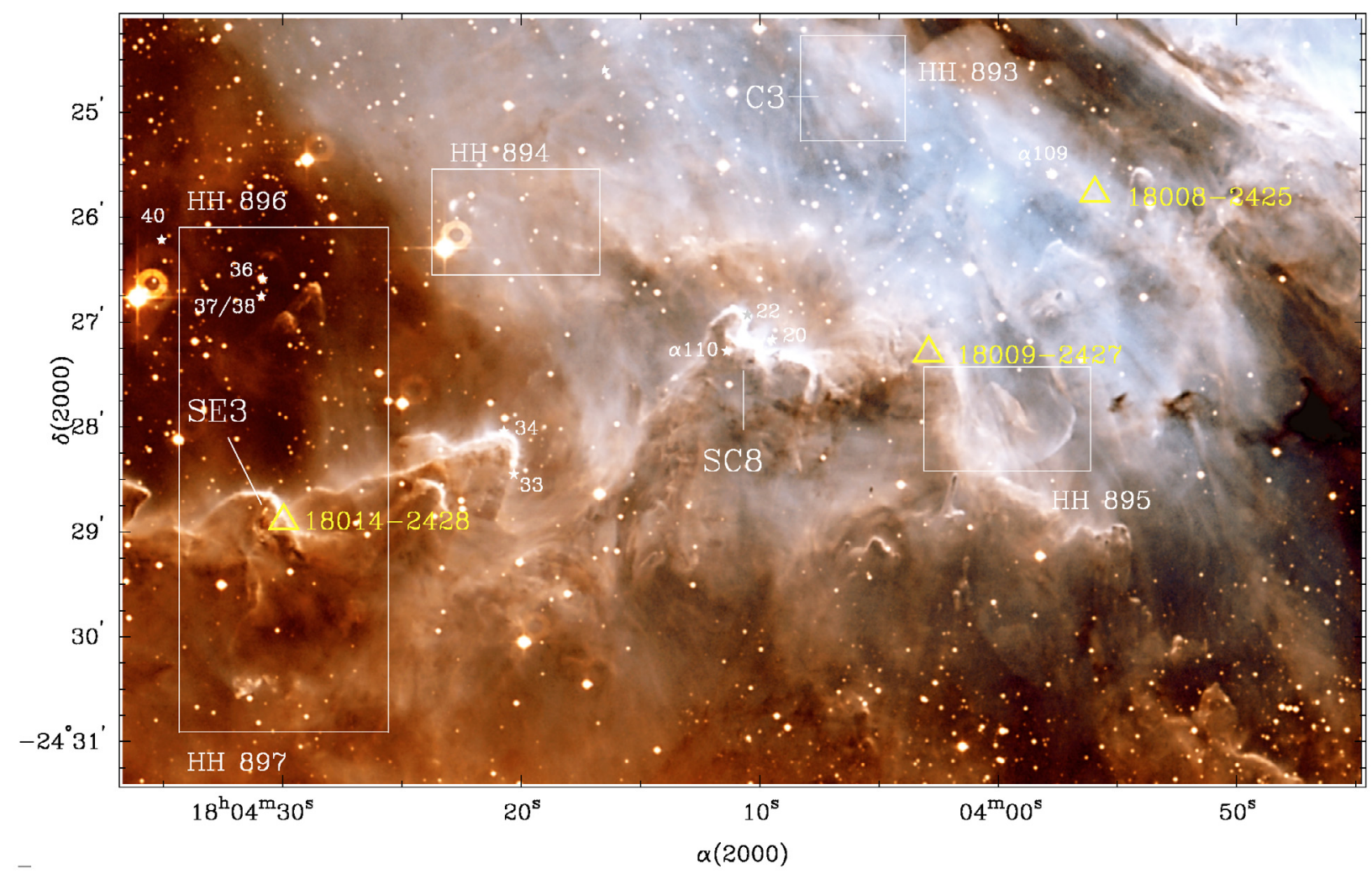

Fig. 1. Three-colour image of the M 8 nebula taken with the WFI at the MPG/ESO 2.2-m telescope, showing [S II] emission (red), H $\alpha$ emission (blue), and $[\mathrm{S} \mathrm{II}]+\mathrm{H} \alpha$ emission (green). The rectangles indicate the regions where the new HH objects are found. Some T Tauri stars identified in the area are denoted with "star" symbols and labelled according to the numbers from Arias et al. (2007). The triangles represent the IRAS sources of interest in this study. The position of the molecular clumps C3, SC8 and SE3 (Tothill et al. 2002) are also marked. The "donuts" next to the bright stars are artifacts due to internal reflections in the filters.
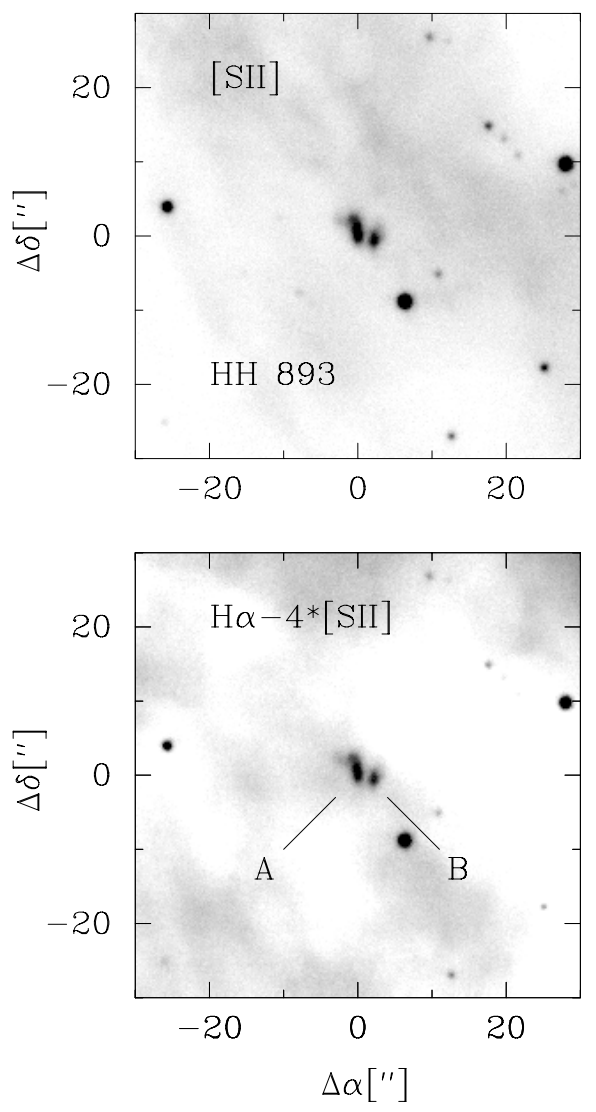
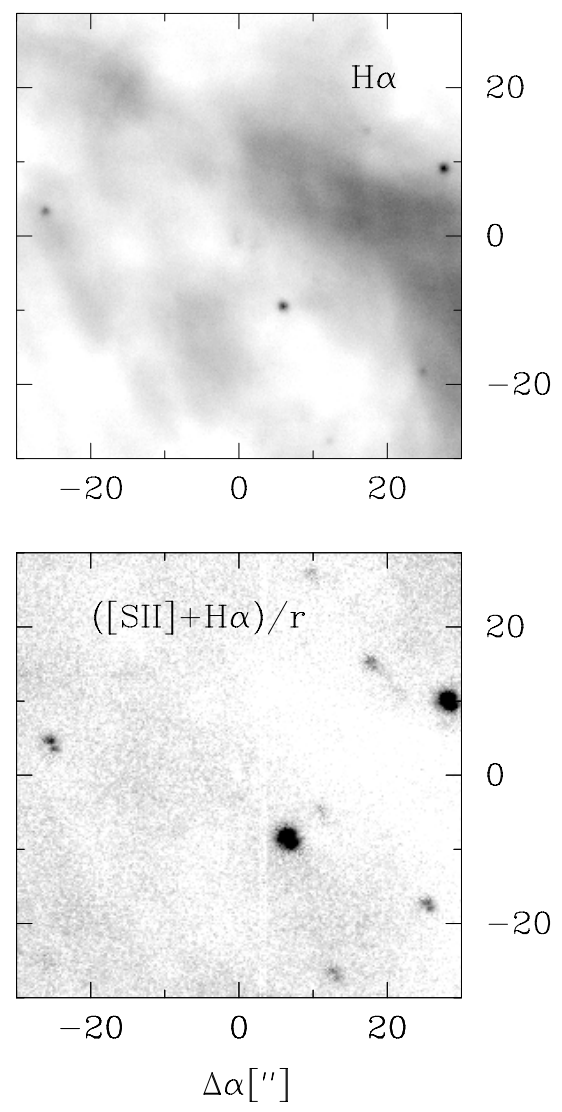

Fig. 2. Four close-ups of HH 893. Top: [S II] (left) and $\mathrm{H} \alpha$ (right) images. Light and dark colours indicate low and high emission values, respectively. Bottom left: difference image obtained by subtracting 4 times the [S II] image from the $\mathrm{H} \alpha$ image. Light colours represent features dominated by $\mathrm{H} \alpha$ emission, and dark colours correspond to features dominated by [S II] emission. Stars appear black because the [S II] image was multiplied by a factor of 4 before subtraction. The labels $\mathrm{A}$ and $\mathrm{B}$, refer to the components described in the text. Bottom right: ratio image between the sum [S II] + $\mathrm{H} \alpha$ and the $R$-band image. In this panel, light colours correspond to features dominated by line emission, while dark colours mean that a continuum light source is present. 


\section{HH 894}

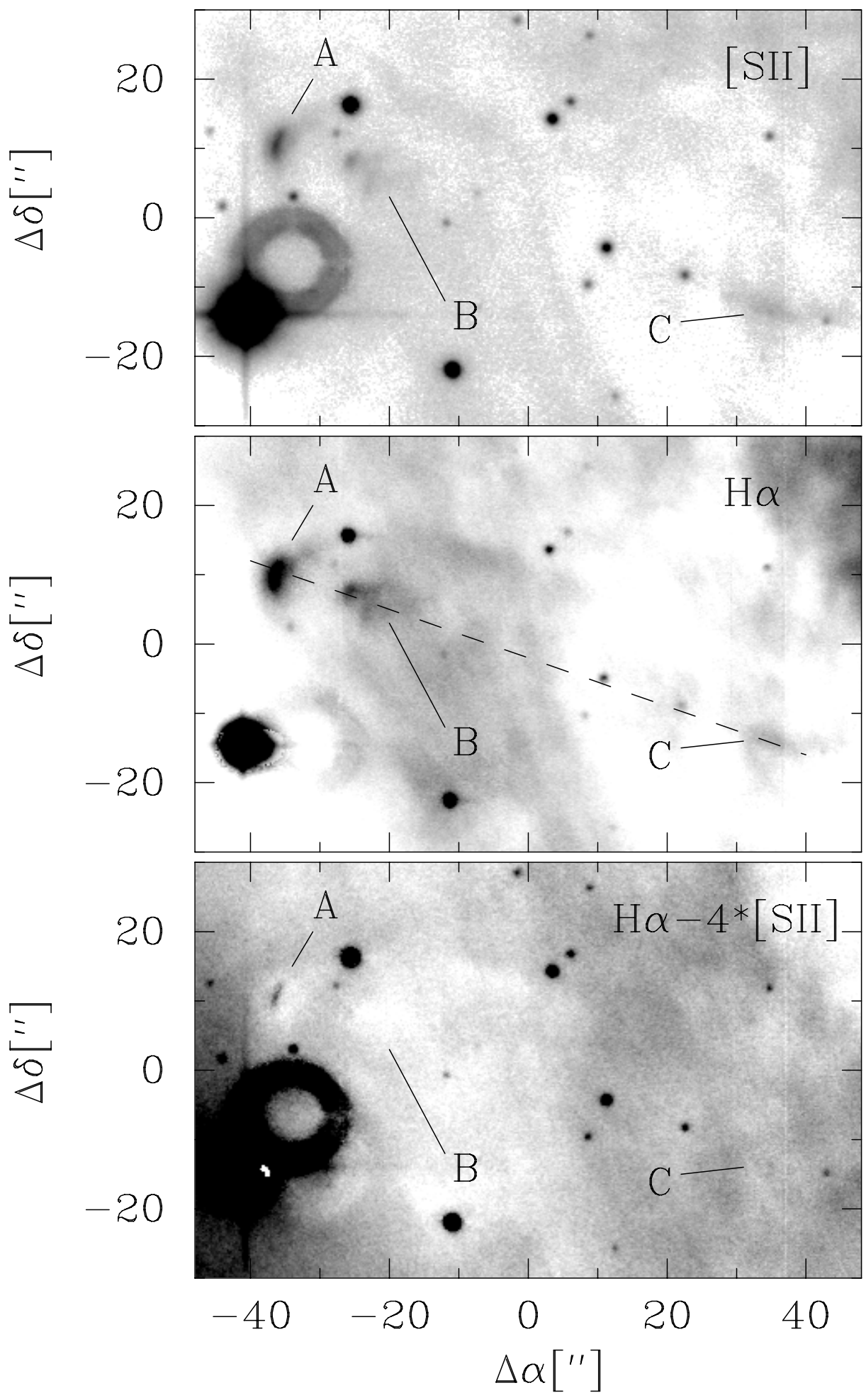

Fig. 3. Three close-ups of HH 894. Top and middle: [S II] and $\mathrm{H} \alpha$ images, respectively. In these panels, light colours indicate low emission values and dark colours correspond to high emission values. The labels A, B and $\mathrm{C}$, refer to the nebular components described in the text. In the $\mathrm{H} \alpha$ image, a dashed line joining these components has been marked. Bottom: difference image obtained by subtracting 4 times the $[\mathrm{S} \mathrm{II}]$ image from the $\mathrm{H} \alpha$ image. Light colours represent features dominated by $\mathrm{H} \alpha$ emission, and dark colours correspond to features dominated by [S II] emission. Stars appear black because the [S II] image was multiplied by a factor of 4 before subtraction. In the three panels, the "donut" next to the bright star on the left is an artifact due to internal reflections in the filters. latter is about $5^{\prime \prime}$ north from the stars ABM 36, ABM 37 and ABM 38, which have been recently classified as T Tauri stars by Arias et al. (2007). The morphologies of the HH $896 \mathrm{~A}$ and B components clearly resemble those observed in the $\mathrm{HH} 1 / 2$ NW and HH 214W objects (Ogura 1995). They are appreciably bright both in $\mathrm{H} \alpha$ and $[\mathrm{S} \mathrm{II}]$ and present a knotty structure, In addition, their axis of symmetry point toward IRAS 18014-2428, although there is a slight difference in their apparent directions.
Perhaps it is premature to give an interpretation regarding the nature of the multiple bow shocks in $\mathrm{HH} \mathrm{896,} \mathrm{but} \mathrm{the} \mathrm{dif-}$ ference in the apparent axis of symmetry of the components A and $\mathrm{B}$ could be related to the phenomenon of jet bending due to the motion of the source within a binary system. This mechanism was proposed by Fendt \& Zinnecker (1998) to explain the misalignment between the apparent direction of propagation for jet and counter jet. Anyway we cannot affirm, based on imaging 


\section{HH 895}

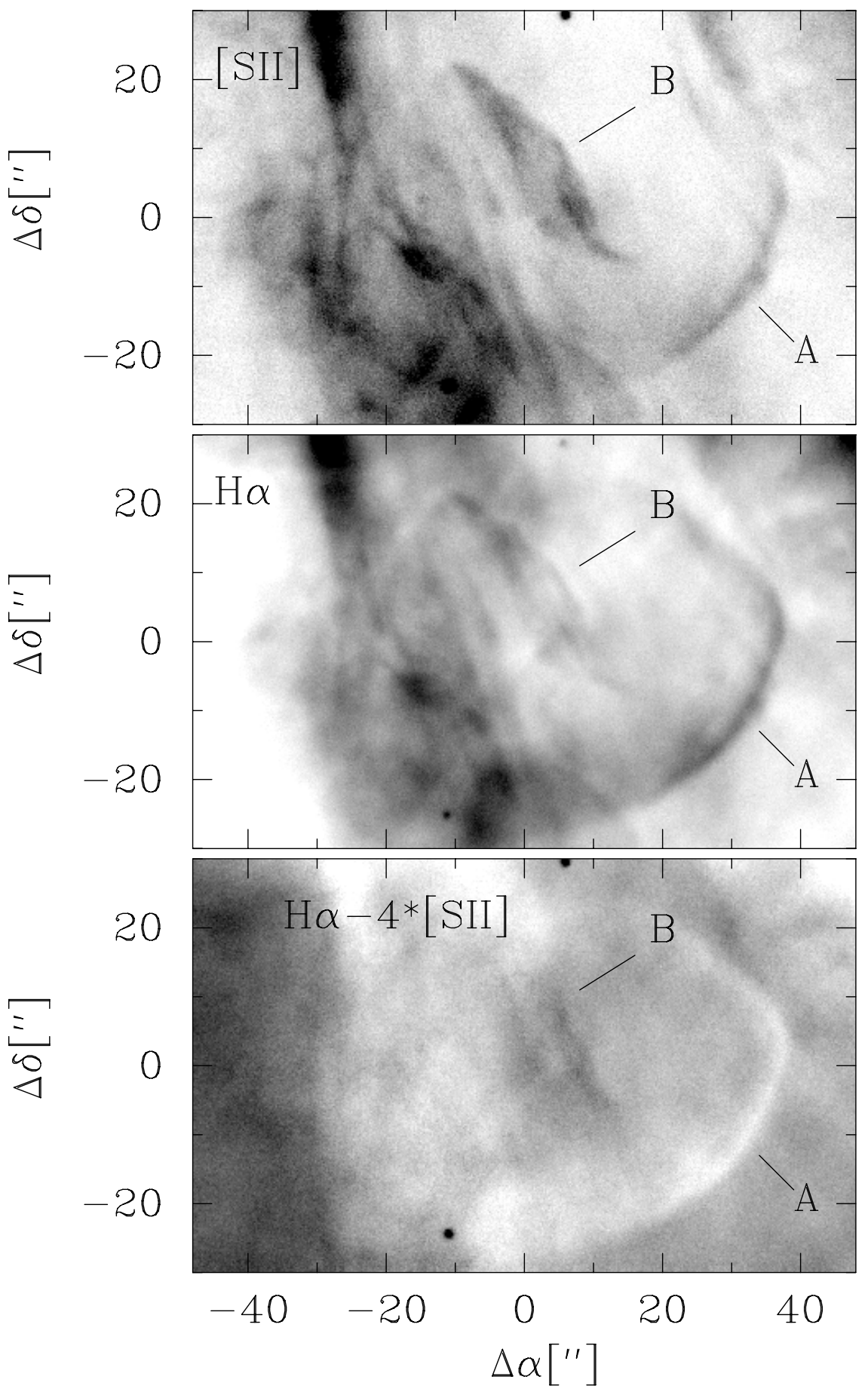

Fig. 4. Three close-ups of HH 895. Top and middle: [S II] and $\mathrm{H} \alpha$ images, respectively. In these panels, light colours indicate low emission values and dark colours correspond to high emission values. The labels A and B, refer to the nebular components described in the text. Bottom: difference image obtained by subtracting 4 times the $[\mathrm{S}$ II] image from the $\mathrm{H} \alpha$ image. Light colours represent features dominated by $\mathrm{H} \alpha$ emission, and dark colours correspond to features dominated by [S II] emission. Stars appear black because the [S II] image was multiplied by a factor of 4 before subtraction. alone, that the bow shocks $\mathrm{A}$ and $\mathrm{B}$ are produced by the two components of a binary system associated with IRAS 18014-2428. Further spectroscopic studies are needed to determine a convincing association between $\mathrm{HH} 896$ and its actual exciting source.

\section{5. $\mathrm{HH} 897$}

HH 897 is situated to the south of the molecular clump SE3 and the IRAS source 18014-2428 (see Figs. 1 and 5). Three nebular emission features (A, B and C) can be identified as subcomponents of HH 897.
Located about 1'.6 south of IRAS 18014-2428, the component A have an irregular knotty morphology. A close-up of this intriguing feature is shown in Fig. 6. There is a pair of nebular structures (B and $\mathrm{C}$ ) connecting the component $\mathrm{A}$ with the mentioned IRAS source. The feature B consist of two parallel filaments, about $14^{\prime \prime}$ long, the western one being somewhat larger. The feature $\mathrm{C}$ is directly associated with the area of IRAS 18014-2428 and the X-ray source WFI 11091 (Prisinzano et al. 2005; Damiani et al. 2004, source 609). It is a rather complex structure that apparently consist of two faint arcs along with several smaller knots. Finally, it is worthwhile to mention that there is a faint "anonymous" stellar object, located 8".5 northeast from 

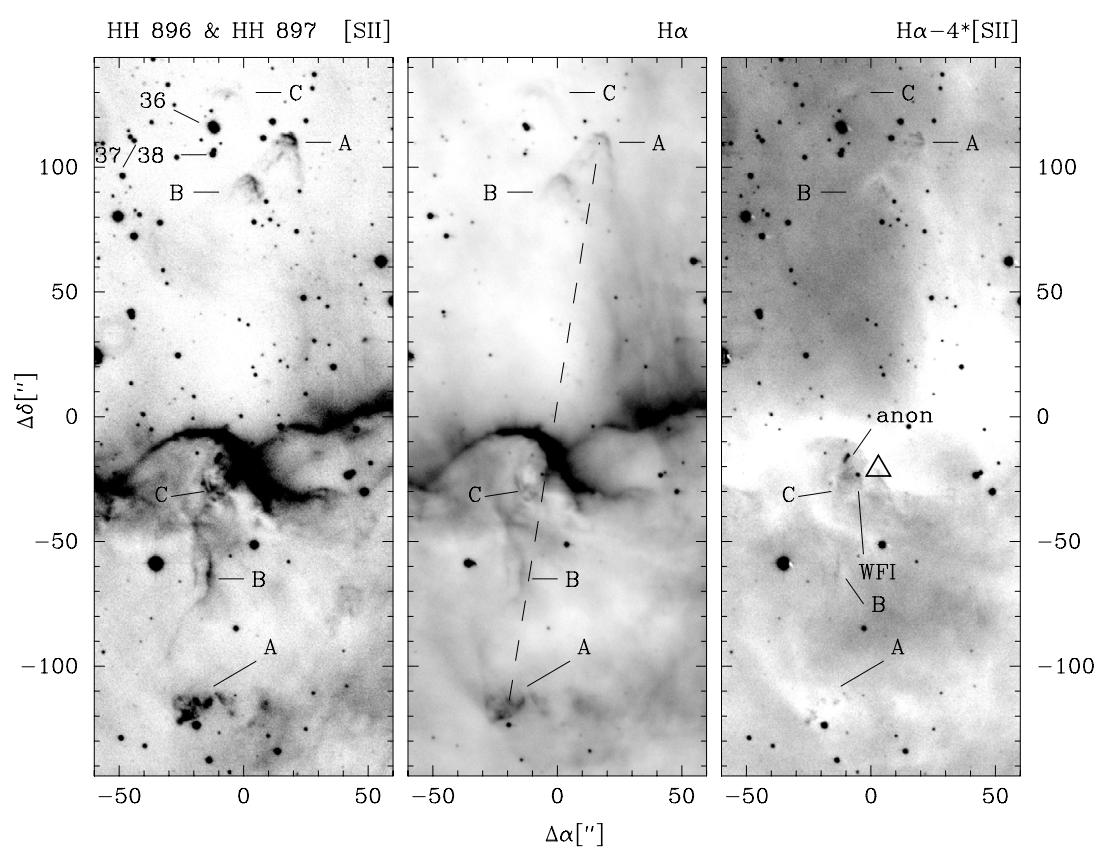

Fig. 5. Three images of the HH 896 and HH 897 outflows. The northern nebular features correspond to HH 896, whereas the southern ones form HH 897. For each case, the labels A, B and C, refer to the subcomponents described in the text. Left: [S II] image. Light colours represent low emission values and dark colours correspond to high emission values. The numbers indicate some T Tauri stars identified by Arias et al. (2007). Middle: $\mathrm{H} \alpha$ image. As in the previous panel, light and dark colours correspond to low and high emission values, respectively. The dashed line represents the presumed axis of the bipolar jet that both $\mathrm{HH}$ objects could be forming (see Sect. 3.6). Right: difference image obtained by subtracting 4 times the [S II] image from the $\mathrm{H} \alpha$ image. Light colours represent features dominated by $\mathrm{H} \alpha$ emission, and dark colours correspond to features dominated by [S II] emission. Stars appear black because the [S II] image was multiplied by a factor of 4 before subtraction. "WFI" and "anon" refer to the two stellar objects found in the $850 \mu \mathrm{m}$ clump SE3 (see text). The triangle marks the position of the IRAS source 18014-2428.
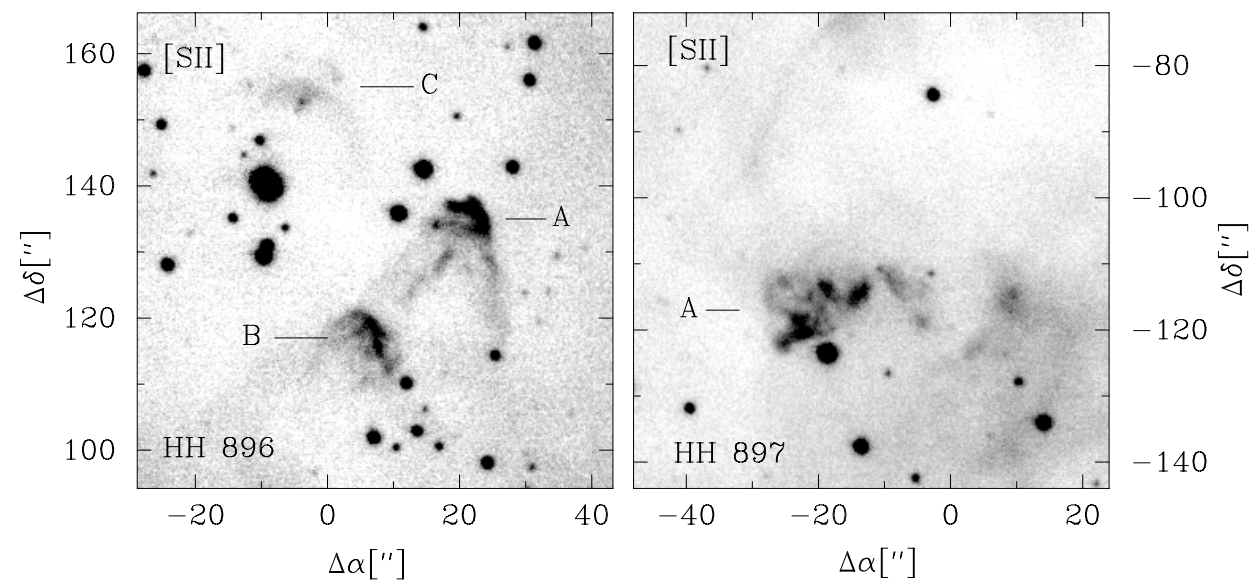

Fig. 6. Close-ups of HH 896 (left) and HH 897 (right) in the light of [S II]. Light and dark colours indicate low and high emission values, respectively. The letters refer to the subcomponents described in the text.

WFI 11091, from which a $\sim 3^{\prime \prime}$ long filament seems to develop $(\mathrm{PA}=150 \mathrm{deg})$.

\subsection{A parsec-scale outflow?}

As described in the previous sections, HH 896 and HH 897 are more or less symmetrically located from the molecular clump SE3 (Tothill et al. 2002) and the IRAS source 18014-2428. It seems fairly likely, based on the observed morphology, that both objects constitute the jet and the counter jet of a single bipolar flow $^{2}$. In Fig. 5, the presumed axis of this bipolar jet has been

\footnotetext{
${ }^{2}$ Spectroscopy recently obtained with Magellan-IMACS (LCO) confirm this conjecture and will be presented in a forthcoming paper.
}

approximately indicated as a dashed line. IRAS 18014-2428 appears as a potential driving source for this outflow. Under this speculation, projected on the sky, the jet formed by HH 896 and HH 897 would have a total length of about 3.9 or roughly $1.4 \mathrm{pc}$ at the distance of M 8, being an example of parsec-scale outflow.

\section{Summary and conclusions}

We report the discovery of five new Herbig-Haro objects in the M 8 nebula at $1.25 \mathrm{kpc}$. These objects, for which the numbers HH 893, HH 894, HH 895, HH 896 and HH 897 have been assigned in the $\mathrm{HH}$ catalogue, show the following characteristics. 
Table 1. New Herbig-Haro objects in M 8 .

\begin{tabular}{clll}
\hline \hline Object & $\alpha_{2000}$ & $\delta_{2000}$ & Notes \\
\hline HH 893 & & & \\
A & 180406.12 & -242446 & {$[\mathrm{SII}]$ knot } \\
B & 180405.95 & -242447 & {$[\mathrm{SII}]$ knot } \\
HH 894 & & & \\
A & 180422.87 & -242552 & parabolic bow-shock \\
B & 180422.01 & -242555 & knots \\
C & 180417.69 & -242616 & filament \\
HH 895 & & & \\
A & 180357.21 & -242804 & wide bow-shock \\
B & 180359.16 & -242753 & knotty filament \\
HH 896 & & & \\
A & 180428.60 & -242638 & parabolic bow-shock \\
B & 180429.75 & -242657 & parabolic bow-shock \\
C & 180430.40 & -242620 & faint bow-shock \\
HH 897 & & & \\
A & 180431.40 & -243027 & knotty bow-shock \\
B & 180431.00 & -242933 & filaments \\
C & 180430.90 & -242859 & arcs and knots \\
\hline
\end{tabular}

$H H$ 893. This is a small feature composed of two nebular emission knots. Its connection with nearby T Tauri stars and/or IRAS sources is rather difficult to discern.

$H H$ 894. This is a peculiar object which consist of three approximately aligned nebular emission features. The presumed axis of this jet intersects the tip of the molecular clump SC8 (Tothill et al. 2002), where the T Tauri stars ABM 20 and ABM 22 (Arias et al. 2007) are located. This leads to the speculation that $\mathrm{HH} 894$ is driven by some of the former young stars, although spectroscopic studies are obviously needed.

$H H$ 895. This is an intriguing object with a very peculiar morphology, located in a region full of filaments and other nebular features.

HH 896. This object consists of three bow-shaped arcs. The faintest one $(\mathrm{C})$ is $\sim 5^{\prime \prime}$ north from a group of recently discovered $\mathrm{T}$ Tauri stars. The other two features (A and B) clearly resemble larger bow shocks commonly seen in HH objects. Their axis of symmetry point toward the IRAS source 18014-2428, located in the molecular clump SE3 (Tothill et al. 2002).

$H H$ 897. This object consist of three emission features with irregular and knotty morphologies. It might be associated with IRAS 18014-2428, as well as with the X-ray source WFI 11091 (Damiani et al. 2004) and another unidentified stellar object present in the clump SE3.

HH 896 and HH 897 are more or less symmetrically located from the molecular clump SE3 and the IRAS source 18014-2428. Based on the observed morphology, it is proposed that these objects constitute the jet and the counter jet of a single parsec-scale bipolar outflow, whose projected total length would be of about 3.9 or roughly $1.4 \mathrm{pc}$ at the distance of M 8. In a forthcoming paper, we will present spectroscopy obtained with Magellan-IMACS (LCO) that confirms this hypothesis.
The IRAS source 18014-2428 appears as a potential driving source for this parsec-scale jet. Molinari et al. (1996) reported an ammonia maser associated with IRAS 18014-2428. They also classified it as a "bonafide" protostar and derived a FIR luminosity of $1.7 \times 10^{4} L_{\odot}$. However the kinematic distance of $2.87 \mathrm{kpc}$ determined for this source might be overestimated, which would imply an overestimation of its luminosity too.

The large projected distances of the objects HH 896 and HH 897 from their potential exciting sources lead to very large dynamical ages. Assuming for the flow speed of the jets a "standard" propagation velocity of $300 \mathrm{~km} \mathrm{~s}^{-1}$, we derive a dynamical age of $4.6 \times 10^{3}$ years for the HH $896 / \mathrm{HH} 897$ system. A future kinematic study through radial velocities and proper motions analysis will certainly help to constrain the numbers suggested here.

The discovery of HH objects in M 8 conclusively demonstrate the existence of very young stars going through the accretion phase of their formation. Finally, we can assert that the presence of large-scale outflows makes the M 8 nebula an especially attractive target for the study of this and other sorts of stellar formation activity.

Acknowledgements. We thank the anonymous referee for many comments and suggestions that have improved this paper. This publication makes use of data products from the Two Micron All Sky Survey, which is a joint project of the University of Massachusetts and the Infrared Processing and Analysis Center/California Institute of Technology, funded by the National Aeronautics and Space Administration and the National Science Foundation. This research has also made use of Aladin and Simbad Database, operated at CDS, Strasbourg, France. Financial support from FONDECYT No. 1050052 and from PIPCONICET No. 5697 is acknowledged by RHB and JIA, respectively. This paper was written during the 10th Workshop of the International Program of Advanced Astrophysics "Guillermo Haro" (INAOE, Tonantzintla, México). The authors are grateful to the organizing committee for the warm and friendly hospitality.

\section{References}

Arias, J. I., Barbá, R. H., Maíz Apellániz, J., Morrell, N. I., \& Rubio, M. 2006, MNRAS, 366, 739

Arias, J. I., Barbá, R. H., \& Morrell, N. I. 2007, MNRAS, 374, 1253

Bally, J., \& Devine, D. 1994, ApJ, 428, L65

Bally, J., Licht, D., Smith, N., \& Walawender, J. 2006, AJ, 131, 473

Comerón, F., \& Reipurth, B. 2006, A\&A, 458, L21

Damiani, F., Flaccomio, E., Micela, G., et al. 2004, ApJ, 608, 781

Fendt, C., \& Zinnecker, H. 1998, A\&A, 334, 750

Goodson, A. P., Winglee, R. M., \& Boehm, K.-H. 1997, ApJ, 489, 199

Hartigan, P., Edwards, S., \& Ghandour, L. 1994, A\&AS, 185, 4815

Hartigan, P., Edwards, S., \& Ghandour, L. 1995, ApJ, 452, 736

Herbig, G. H. 1957, ApJ, 125, 654

Kastner, J. H., Franz, G., Grosso, N., et al. 2005, ApJS, 160, 511

Lada, C. J., Gull, T. R., Gottlieb, C. A., \& Gottlieb, E. W. 1976, ApJ, 203, 159

Molinari, S., Brand, J., Cesaroni, R., \& Palla, F. 1996, A\&A, 308, 573

Ogura, K. 1995, ApJ, 450, L23

Prisinzano, L., Damiani, F., Micela, G., \& Sciortino, S. 2005, A\&A, 430, 941

Reipurth, B. 1981, A\&AS, 44, 379

Reipurth, B., Heathcote, S., \& Vrba, F. 1992, A\&A, 256, 225

Reipurth, B., \& Bally, J. 2001, ARA\&A, 39, 403

Skrutskie, M. F., Cutri, R. M., Stiening, R., et al. 2006, AJ, 131, 1163

Stecklum, B., Launhardt, R., Fischer, O., et al. 2004, ApJ, 617, 418

Tothill, N. F. H., White, G. J., et al. 2002, ApJ, 580, 285

Turner, N. J., Bodenheimer, P., \& Rozyczka, M. 1999, ApJ, 524, 12

White, R. J., \& Hillenbrand, L. A. 2004, ApJ, 616, 998

Zhang, Q., Hunter, T. R., Brand, J., et al. 2005, ApJ 625, 864 